\title{
Maternal Supplementation with $R R R$ - $\alpha$-Tocopherol (400 IU) and Its Relationship with Serum and Breast Milk Retinol
}

\author{
Juliana Fernandes dos Santos Dametto ${ }^{1}$, Larissa Queiroz de Lira ${ }^{2}$, Larisse Rayanne \\ Miranda de Melo ${ }^{1}$, Marina Mendes Damasceno ${ }^{1}$, Nathalia Lorena do Nascimento \\ Silva ${ }^{1}$, Roberto Dimenstein ${ }^{2}$ \\ ${ }^{1}$ Department of Nutrition, Federal University of Rio Grande do Norte, Natal, RN, 59078- \\ 970, Brazil; julianadametto.dnut@ufrnet.br (J.F.S.D.); larissermm@hotmail.com \\ (L.R.M.M.); marina.mendesnutri@gmail.com (M.M.D.); natx_lorena@hotmail.com \\ (N.L.N.). \\ ${ }^{2}$ Department of Biochemistry, Federal University of Rio Grande do Norte, Natal, RN, \\ 59072-970, Brazil; liralq@gmail.com (L.Q.L.); rdimenstein@gmail.com (R.D.). \\ *Correspondence: julianadametto.dnut@ufrnet.br; Tel.: +55-84-999143973
}

\begin{abstract}
Vitamin A and E are important during pregnancy, the neonatal period, and childhood. The objective of this study was to assess whether maternal $R R R$ - $\alpha$-tocopherol supplementation affects serum and breast milk retinol. Serum was collected at baseline and twenty days later, and breast milk, at baseline, and on days 1, 7, and 20 after delivery. After the baseline serum collection, the supplemented group $(n=16)$ received a single 400 IU of $R R R$ - $\alpha$-tocopherol. The control group $(\mathrm{n}=18)$ was only performed collections. Retinol and alpha tocopherol levels were determined by liquid chromatography. Serum retinol and alpha tocopherol at baseline and 20 days after delivery indicated proper vitamin $\mathrm{A}(>20 \mu \mathrm{g} / \mathrm{dL})$ and $\mathrm{E}(>516 \mu \mathrm{g} / \mathrm{dL})$ statuses in the control and supplemented groups $(\mathrm{p}>0.05)$. Colostrum retinol levels on days 1 and 7 after delivery were significantly higher in the supplemented group $(\mathrm{p}=0.018$ and $\mathrm{p}=0.012$, respectively). Maternal vitamin E supplementation increased colostrum retinol by $52.23 \%$ and $111.2 \%$, 24 hours and 7 days, respectively. However, retinol in mature milk did not differ between the groups $(\mathrm{p}>0.05)$. In conclusion, the supplementation with 400 IU of $R R R-\alpha-$ tocopherol improved vitamin A bioavailability in breast milk.
\end{abstract}

Keywords: vitamin E; vitamin A; maternal serum; lactation; liquid chromatography.

\section{Introduction}


Vitamin A is an essential micronutrient for many metabolic processes, and it is especially important during times of rapid cell proliferation and differentiation, such as pregnancy, the neonatal period, and childhood . This vitamin acts as a carrier of mannosyl residues, in the synthesis of some glycoproteins, and in the production of mucus and resistance to infections mediated by the modulating action of cell growth and differentiation [1].

The term vitamin $\mathrm{A}$ is generically used for all beta-ionone derivatives that have the biological activity of all-trans retinol or that are structurally correlated to it, except retinoids, which are called provitamin A [2,3].

This vitamin is present in some foods in two forms: preformed vitamin A and provitamin A of the carotenoids. Preformed vitamin A is present in animal foods, such as eggs, liver, and dairy products. Provitamin A is present in green and yellow vegetables, and in some orange fruits [2-4].

Vitamin A deficiency can lead to xerophthalmia and night blindness in children, to anemia, and to low resistance to infections, which can increase the severity of infectious diseases and the risk of death [2,3]. Preschoolers and pregnant women are the populations at greatest risk of vitamin A deficiency [5].

Vitamin E also has an important role in the health of newborns. Like vitamin A, it is a fat-soluble vitamin, and its main function is its antioxidant activity in cell membranes as it can inhibit lipid peroxidation [6]. This vitamin has eight chemical compounds, namely $\alpha-, \beta-, \gamma$-, $\delta$-tocopherol, and tocotrienols, but these compounds have different structures and bioavailability. Alpha-tocopherol is the most active form. The other forms to not contribute to the nutritional requirements of vitamin $\mathrm{E}$ [4].

Alpha-tocopherol is an efficient inhibitor of lipid peroxidation in vivo as it donates a hydrogen to the peroxyl radical, interrupting chain reactions and consequently, protecting the long-chain polyunsaturated fatty acids and lipoproteins in cell membranes from oxidation; it is the main fat-soluble antioxidant in tissues [6].

Preterm infants are born with sparse body fat and low body stores of fat-soluble vitamins, including vitamin E [7]. Newborns are considered a high-risk group for vitamin E deficiency. Insufficient consumption of vitamin $\mathrm{E}$ at this phase of life can compromise the immune system and affect lung development [8]. However, giving extra vitamin E to preterm babies can provide some benefits, but it increases the risk of life-threatening infections. Preterm babies (born before 37 weeks) can develop a range of problems because their organs are not mature. Vitamin E may be able to help prevent or limit some 
of these problems, but it can potentially also have harmful effects [9]. The main source of alpha tocopherol for newborns is breast milk. Alpha tocopherol prevents possible damage from oxidative stress generated by the transition from a prenatal intrauterine environment relatively poor in oxygen to a postnatal extra uterine environment considerably richer in oxygen. Maternal supplementation with alpha tocopherol can be an efficient way to boost vitamin E levels in neonates [8]

Vitamins A and E have critical roles on the satisfactory health and development of newborns. Yet, fetal reserves of vitamins A and E are limited possibly because of placental barrier selectivity, which regulates the passage of both vitamins from the mother to the fetus, probably to avoid teratogenic effects. Hence, breast milk is the only source of these nutrients for exclusively breastfed children. Breast milk provides antioxidant protection and stimulates the newborn's immune system development [10].

Some mammal, including human, studies have found that vitamin A supplementation can reduce serum and/or breast milk $\alpha$-tocopherol because these vitamins share some tissue transport and distribution pathways $[5,11,12]$. On the other hand, [13] found that fat-soluble vitamins are absorbed in different sites in rats: vitamin $\mathrm{A}$ is more widely absorbed in the proximal region, and vitamin $\mathrm{E}$, in the distal region of the small intestine. Moreover, the same study found that when vitamins A and $\mathrm{E}$ are taken together, some vitamin $\mathrm{E}$ is degraded, which reduces its absorption, and vitamin $\mathrm{E}$ also protects vitamin A from oxidation.

The purpose of this study was to assess whether maternal supplementation with RRR- $\alpha$-tocopherol (400 IU) affects serum and breast milk retinol. The null hypothesis was that breast milk and serum vitamin A levels were not influenced by vitamin $\mathrm{E}$ supplementation.

\section{Materials and Methods}

\subsection{Participants}

This prospective, controlled, and randomized study included two groups of volunteers recruited at the School Maternity Hospital Januário Cicco and Unidade Mista das Quintas, both in Natal-RN, Brazil. These institutions were chosen because they provide obstetric services for women from Natal and other municipalities in the state of Rio Grande do Norte. 
Data and samples were collected from March 2016 to March 2017, and the women were followed for 20 days after delivery. The study was approved in 07 March 2016 by the Research Ethics Committee of the Federal University of Rio Grande do Norte (UFRN) under protocol number 1.441.203 CAAE 43894615.4.0000.5537, complied with the Declaration of Helsinki guidelines, and was registered at ReBEC (Brazilian Registry of Clinical Assays) under code number RBR-9wch5m. The study is available at: http://www.ensaiosclinicos.gov.br/rg/?q=RBR-9wch5m.

The sample size was calculated by the software GPower ${ }^{\circledR}$ version 3.1.9.2 [14] based on the following parameters: $\alpha$ of $5 \%$; power of 0.8 ; and expected effect of 0.40 . Hence, each group should have at least twelve individuals.

The participants were informed about the objectives of the study and signed an informed consent form before enrollment. The inclusion criteria were age between 18 and 40 years, low obstetric risk, and term delivery (gestational age equal to or greater than 37 weeks). The exclusion criteria were: use of multivitamins during pregnancy and/or lactation, maternal complications or acute/chronic diseases, preterm delivery (gestational age $<37$ weeks), fetal malformation, and/or multiple pregnancies.

The first woman to deliver was allocated to the control group (CG), the second to the supplemented group (SG), the third to the CG, and so on. This study was organized as recommended by the Consolidated Standards of Reporting Trials - CONSORT (Figure $1)$.

The CG did not receive any intervention. The supplemented group received a single 400 IU capsule of $R R R$ - $\alpha$-tocopherol (d- $\alpha$-tocopherol acetate, gelatin (bovine), soybean oil, glycerin) on the first day after delivery and after collection of the biological material. The supplement was taken orally with water.

A $400 \mathrm{IU}$ dosage of $R R R$ - $\alpha$-tocopherol corresponds to $268.5 \mathrm{mg}$ of $\alpha$-tocopherol. This dosage was chosen because it has been considered safe by the Institute of Medicine [15] after analysis of long-term studies, which found no deleterious effects associated with this dosage. 


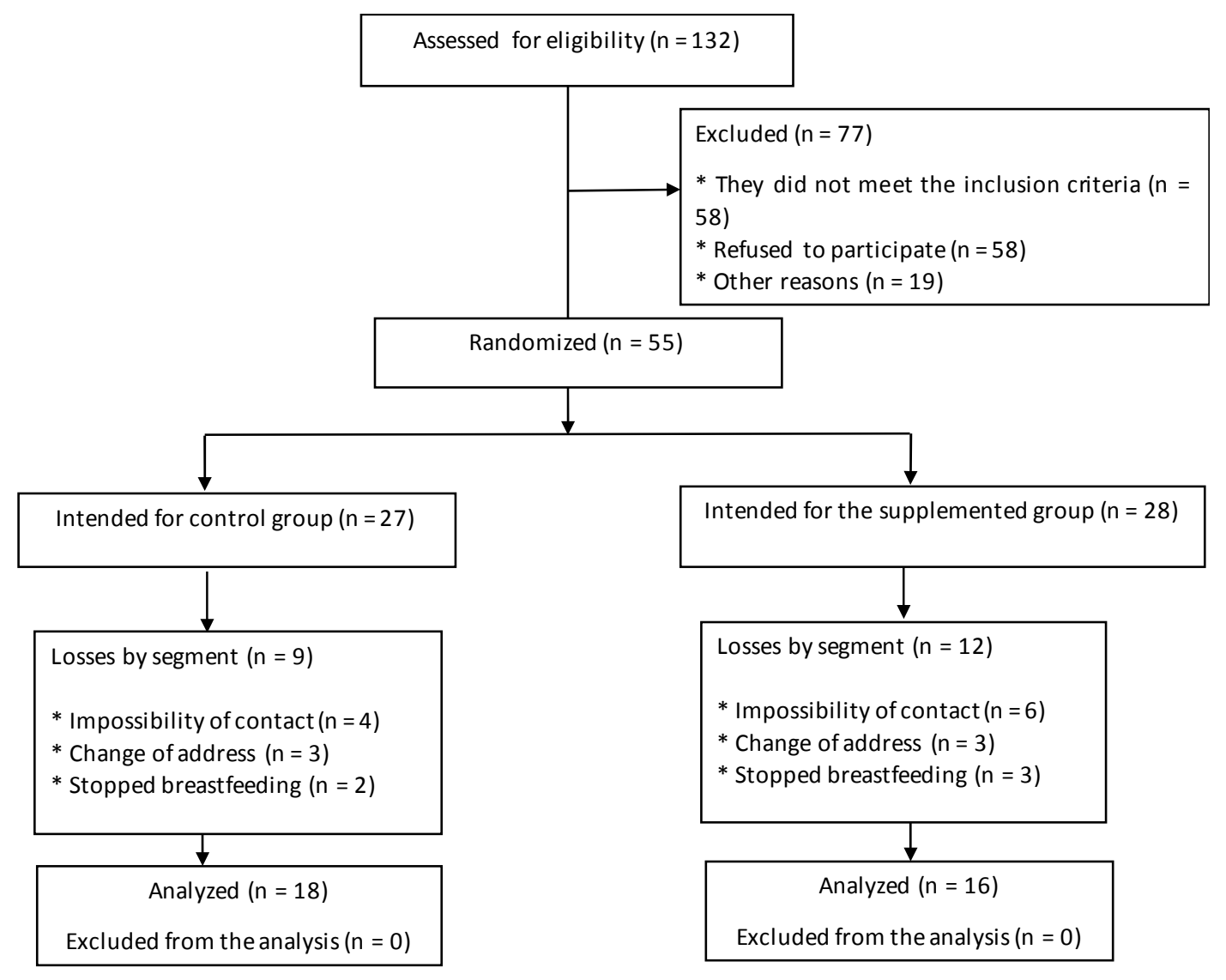

Figure 1 - Consolidated Reporting Trials (CONSORT) flow diagram.

\subsection{Collection of data and biological material}

Socioeconomic, prenatal, and delivery data, clinical history, and biochemical tests were collected from the patients' medical records.

Blood and breast milk were also collected. On the first day after delivery, immediately after collection of five milliliters of blood and two milliliters of breast milk from all participants, the participants in the SG received a 400 IU capsule of $R R R-\alpha-$ tocopherol. No intervention was done on the CG. On the second day after delivery, two milliliters of breast milk were collected from all participants. On the seventh day after delivery, two milliliters of breast milk were collected from all participants, and on the twentieth days after delivery, five milliliters of blood were collected from all participants. All collections were done in the morning after an overnight fast. Of the 55 study women, 34 completed the study, 18 in the CG and 16 in the SG.

The biological materials were collected and stored in opaque polypropylene tubes, which were immediately refrigerated and delivered to the Laboratory of Food and 
Nutrition Biochemistry of UFRN. The blood samples were centrifuged at $4000 \mathrm{rpm}$ for 10 minutes to separate the serum. Maternal serum and milk were stored at $-20{ }^{\circ} \mathrm{C}$ for zero to four days until analysis.

\subsection{Biochemical analyses}

Serum $\alpha$-tocopherol and retinol were extracted as recommended by Ortega et al. [16]. One $\mathrm{mL}$ of $95 \%$ ethanol (Merck, Darmstadt, Germany) and $2 \mathrm{~mL}$ of hexane (Merck) were added to $1 \mathrm{~mL}$ of serum.

Later, the samples were homogenized for one minute and centrifuged for ten minutes to separate the hexane phase. This stage was repeated three times, totaling $6 \mathrm{~mL}$ of hexane phase. Two $\mathrm{mL}$ of hexane phase were collected from each sample and the hexane was evaporated in a water bath at $37^{\circ} \mathrm{C}$. The extract was then diluted with $250 \mu \mathrm{L}$ of absolute ethanol (Vetec, Speyer, Germany), and $20 \mu \mathrm{L}$ were injected in the highperformance liquid chromatography (HPLC) device.

Breast milk retinol was extracted as recommended by Giuliano et al. [17]. Five hundred $\mathrm{mL}$ of $95 \%$ ethanol and $500 \mu \mathrm{L}$ of $50 \% \mathrm{w} / \mathrm{v}$ potassium hydroxide were added to $500 \mu \mathrm{L}$ of breast milk. The mixture was vortexed for one minute, placed in a water bath at $60^{\circ} \mathrm{C}$ for one hour, and stirred at 10 -minute intervals. Two $\mathrm{mL}$ of hexane was added to the mixture, and the mixture was again vortexed for one minute and centrifuged $(500 \mathrm{~g})$ for 10 minutes. The hexane phase was then transferred to another tube. This process was repeated three times. Aliquots of $3 \mathrm{~mL}$ of hexane were collected from the baseline and on days 1, 7 and 20 breast milk samples. These aliquots were evaporated in a water bath at $37^{\circ} \mathrm{C}$. The extract was diluted with $250 \mu \mathrm{L}$ of absolute ethanol, and $20 \mu \mathrm{L}$ were injected in the HPLC device.

Retinol was extracted from mature milk as recommended by Giuliano et al. [17], described above. However, only one $\mathrm{mL}$ of breast milk was extracted, so only one $\mathrm{mL}$ of $95 \%$ ethanol (Merck) and one $\mathrm{mL}$ of $50 \% \mathrm{w} / \mathrm{v}$ potassium hydroxide were added to it. The dry extract was then diluted with $250 \mu \mathrm{L}$ of absolute ethanol.

The amounts of $\alpha$-tocopherol and retinol in the biological samples were measured by the chromatographic device Shimadzu (Shimadzu Corp., Kyoto, Japan). This device contains the pump LC-10 AD Shimadzu coupled to the detector SPD-10A Shimadzu UVVIS, the column CLC-ODS (M) 4.6 x 250 mm (Perkin Elmer, Boston, MO, USA), and the integrator Chromatopac C-R6A Shimadzu. The isocratic mobile phase in all analyses 
consisted of $100 \%$ methanol flowing at $1.0 \mathrm{ml} / \mathrm{min}$. The wavelengths used for quantifying $\alpha$-tocopherol and retinol were $292 \mathrm{~nm}$ and $325 \mathrm{~nm}$, respectively.

Alpha-tocopherol and retinol in the samples were identified and quantified by comparing the retention time and area provided by the chromatographic profile with standard stock solutions of $\alpha$-tocopherol and retinol, respectively (Sigma, St Louis, MO, USA). The concentrations of the standards were confirmed by the specific extinction coefficients in absolute ethanol for $\alpha$-tocopherol $(\varepsilon 1 \%, 1 \mathrm{~cm}=75.8$ at $292 \mathrm{~nm})$ and retinol $(\varepsilon 1 \%, 1 \mathrm{~cm}=1,780$ at $325 \mathrm{~nm})[18,19]$.

Maternal vitamin E deficiency was defined as serum $\alpha$-tocopherol $<516 \mu \mathrm{g} / \mathrm{dL}$ $(12 \mu \mathrm{mol} / \mathrm{L})$, the cut-off point for healthy adults [15]. A cut-off point for adequate vitamin $\mathrm{E}$ in breast milk does not exist.

Maternal vitamin A deficiency (VAD) was defined as serum retinol $<20 \mu \mathrm{g} / \mathrm{dL}$ $(0.70 \mu \mathrm{mol} / \mathrm{L})[20]$, and low serum retinol was defined as serum retinol between 20 and $30 \mu \mathrm{g} / \mathrm{dL}(0.70$ and $1.05 \mu \mathrm{mol} / \mathrm{L})$ [21].

Low colostrum retinol was defined as colostrum retinol $\leq 60 \mu \mathrm{g} / \mathrm{dL}(2.1 \mu \mathrm{mol} / \mathrm{L})$ [22], and low mature milk retinol was defined as mature milk retinol $<30 \mu \mathrm{g} / \mathrm{dL}(1.05$ $\mu \mathrm{mol} / \mathrm{L})[20]$.

\subsection{Statistical analyses}

The statistical analyses were performed by the software IBM SPSS version 21.0 for Windows (SPSS Inc., Chicago, IL, USA). Serum and breast milk $\alpha$-tocopherol and retinol were expressed as means, standard deviations, and percent frequency. The Shapiro-Wilk test confirmed that all numerical variables had normal distribution. Group homogeneity was verified by the chi-square test. The Student's t-test for independent samples compared the serum vitamins between the groups, and the Levene test verified intergroup variance homogeneity. The Student's t-test for paired samples tested the difference between intragroup vitamin levels. The significance level was set at $5 \%$ (p < $0.05)$.

\section{Results}

Among 132 women recruited before delivery to participate in the study, 77 were excluded due to lack of milk or blood data, and 55 were included in the characterization, 27 of the control group and 28 of the supplemented group. 
Table 1 presents the maternal, obstetric and neonatal characteristics. The participants had a mean educational level, 23 (46\%) had monthly income between 1 and 3 minimum wages, 31 (56\%) had normal birth and 24 (47\%) were overweight or obese at the end of gestation. None of the characteristics mentioned above were associated with serum retinol $(\mathrm{p}>0.05)$, and the groups were homogeneous.

Table 1 - Characterization of the study population based on maternal and newborn variables.

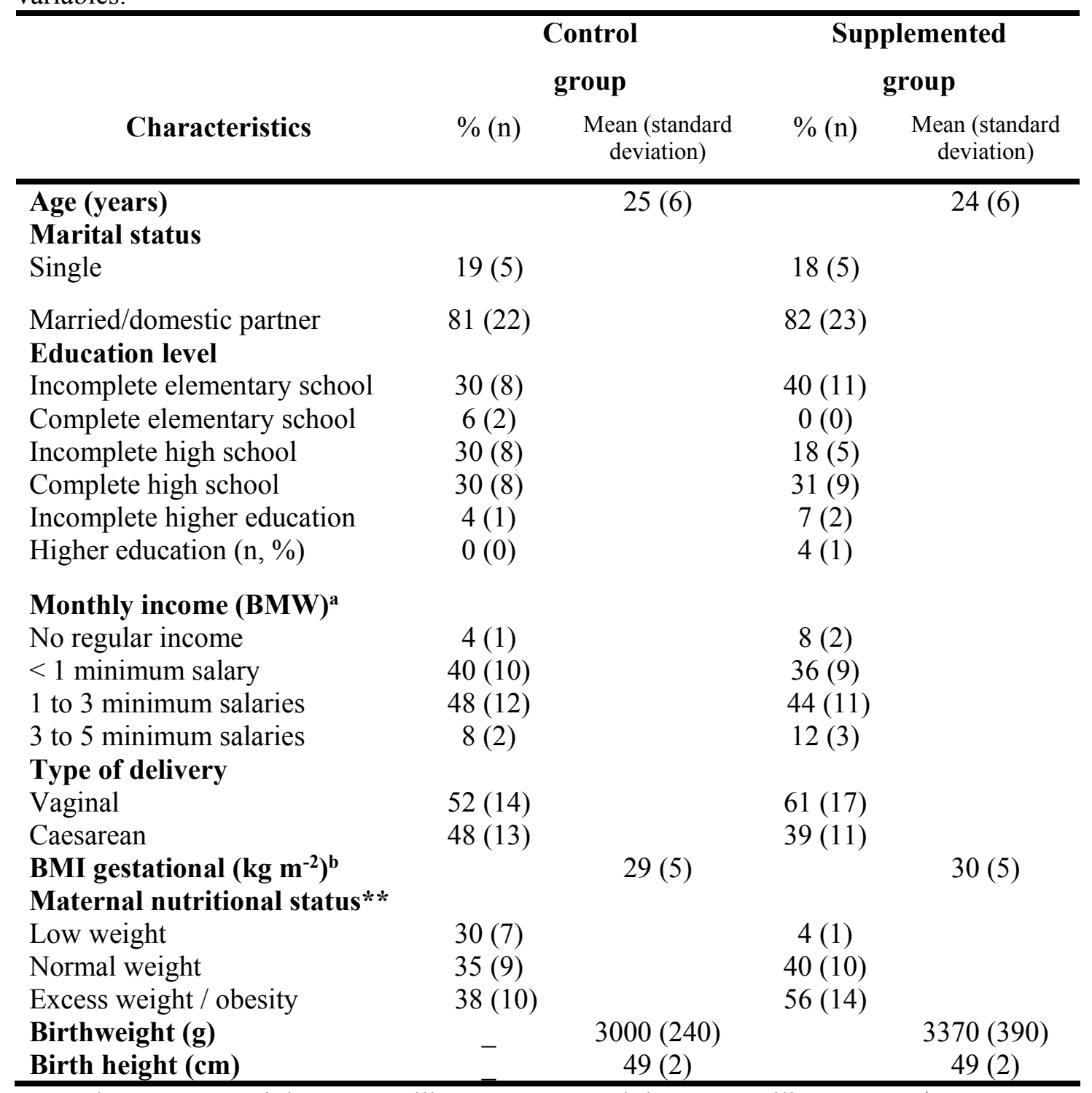

n, number; ${ }^{a}$ BMW, minimum Brazilian wage; One minimum Brazilian wage $=\$$ US 372; bBMI, body mass index; BMI / gestational week (Atalah et al., 1997). 
In the biochemical analysis 34 women participated in the study, 18 of the control group and 16 of the supplemented group. Baseline serum $\alpha$-tocopherol $(\mu \mathrm{g} / \mathrm{dL})$ and retinol $(\mu \mathrm{g} / \mathrm{dL})$ of the control group were $1195 \pm 328$ and $44 \pm 14$, respectively, and on day 20, $908 \pm 439$ and $43 \pm 12$, respectively. Baseline serum $\alpha$-tocopherol $(\mu \mathrm{g} / \mathrm{dL})$ and retinol $(\mu \mathrm{g} / \mathrm{dL})$ of the supplemented group were $1184 \pm 411$ and $42.5 \pm 13$, respectively, and on day 20, $956 \pm 381$ and $42.2 \pm 12$, respectively. Those levels did not differ between the groups on any occasion $(\mathrm{p}>0.05)$.

Both groups had appropriate serum levels of $\alpha$-tocopherol and retinol according to the established cut-off points.

Colostrum retinol levels in the control group were: $114.3 \pm 60 \mu \mathrm{g} / \mathrm{dL}$ at baseline; $108 \pm 66 \mu \mathrm{g} / \mathrm{dL}$ on day 01 after delivery; $38.5 \pm 19.5 \mu \mathrm{g} / \mathrm{dL}$ on day 07 after delivery; and $53 \pm 36.3 \mu \mathrm{g} / \mathrm{dL}$ in mature milk. Colostrum retinol levels in the supplemented group were: $110 \pm 50.4 \mu \mathrm{g} / \mathrm{dL}$ at baseline; $167.3 \pm 57 \mu \mathrm{g} / \mathrm{dL}$ on day 01 after delivery; $81.3 \pm 51.3$ $\mu \mathrm{g} / \mathrm{dL}$ on day 07 after delivery; and $43 \pm 13.2 \mu \mathrm{g} / \mathrm{dL}$ in mature milk.

The main significant effect appeared when breast milk retinol levels were compared over time $(\mathrm{p}<0.01, \eta 2=0.544)$, but a significant effect was not found between the groups $(p=0.506, \eta 2=0.032)$. A significant effect was found when the interaction between the factors 'breastfeeding duration' and 'study groups' was analyzed $(\mathrm{p}<0.01$, $\eta 2=0.279$ ). Thus, the colostrum retinol of the supplemented group had increased by $52.23 \% 24$ hours after the intervention. When the two groups were compared on day 07 after the intervention, the breast milk retinol of the supplemented group was $111.17 \%$ higher than that of the control group (Figure 2).

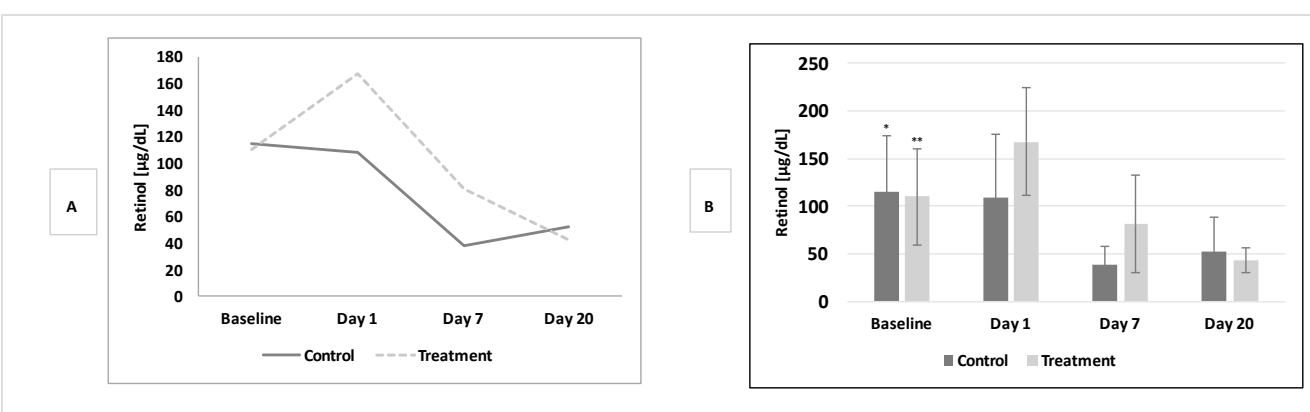

Figure 2. Retinol profile from baseline (immediately after delivery) to 20 days after delivery in the control and treatment groups. Natal/RN, 2016-2017. A - postnatal effect of breast milk retinol. Split-plot ANOVA: $p<0.01, \eta 2=0.544$. 
$\mathrm{B}-*$ breast milk retinol difference between baseline and day $07(\mathrm{p}=0.001)$; between day 01 and day 07

$(\mathrm{p}=0.008)$; between baseline and day $20(\mathrm{p}<0.01)$; and between day 01 and day $20(\mathrm{p}=0.003)$.

** breast milk retinol difference between baseline and day $01(\mathrm{p}=0.008)$; between baseline and day $20(\mathrm{p}$

$<0.01)$; between day 01 and day $07(\mathrm{p}<0.01)$; between day 01 and day $20(\mathrm{p}<0.01)$; and between day 07 and day $20(\mathrm{p}=0.006)$. One-way ANOVA.

\section{Discussion}

A balanced diet during pregnancy is necessary to guarantee the availability of nutrients to the fetus, thereby preparing the fetus for birth and breastfeeding. All nutrients are essential during this period, but vitamin A stands out because of its important role in the cell growth and proliferation that occurs during pregnancy, breastfeeding, and early childhood [23].

Micronutrient deficiency during pregnancy may cause numerous adversities for maternal and fetal health. During lactation, nutritional deficiencies may contribute to infants' low nutrient reserves, thus increasing the prevalence of nutritional deficiencies in the first years of life [24].

The two study groups consisted of adult women who were normal weight at conception and birthed normal birth weight infants, confirming other studies in similar populations $[4,25]$.

Serum $\alpha$-tocopherol and retinol levels in both groups indicated that both groups had proper vitamin E and A statuses, respectively. The baseline serum alpha tocopherol levels of the study control and supplemented groups were similar to the levels reported by other studied similar populations [4,26,27]. However, the study results are higher than those reported by Rodriguez et al.[28], but the difference may stem from the population studied by the latter, namely, a population living in poverty with terrible health indicators. The alpha tocopherol levels of the study groups were lower than those reported by Garcia et al. [11] and Dimenstein et al. [29], which also may be justified by their samples, consisting of adult and adolescent women.

The baseline serum retinol levels of the study control and supplemented groups are similar to those reported by Fernandes et al. [30]; Lima et al. [10] and Grilo et al. [25], who studied women from the Brazilian northeast region. However, the baseline serum retinol levels of the study control and supplemented groups were higher than those found in India [31], Africa, [32,33], and Ethiopia [34] as these countries have high prevalence of vitamin A deficiency, and in Germany [35], probably because of the very different 
sample size. On the other hand, Yang et al. [36] reported a higher level of serum retinol possibly because of different diets of the two samples and sampling methods.

Breast milk is the best food for newborns because of its ideal nutritional characteristics, high levels of fat-soluble vitamins [37], and ability to provide important immune and psychological factors that reduce infant morbidity and mortality [38]. Some studies have suggested that colostrum may be a biochemical indicator of vitamin A status; therefore, breast milk vitamin A can be used for monitoring the impact of vitamin Arelated intervention programs as it is easy to obtain in a non-invasive manner [37,39].

The baseline colostrum retinol levels in the study control and supplemented groups were higher than those reported in Cuba [22], where women have very bad health, and in Brazil [25] possibly because of different sample sizes. The study retinol level was lower than that reported in Germany [37], where people have better access to food and counseling during pregnancy and puerperium.

Countries with high risk of vitamin A deficiency have a supplementation policy that consists of giving mothers a megadose of vitamin A (200,000 IU) at most six weeks after delivery to increase the level of this vitamin in breast milk (World Health Organization 2009). Study [25] have assessed the action of vitamin A on vitamin E, and the present study investigated for the first time whether maternal supplementation of vitamin E after delivery increases retinol, which it does as colostrum retinol 24 hours after vitamin E supplementation increased and remained high until the seventh day after supplementation.

Moore [40] found that vitamin $\mathrm{E}$ in the diet of rats increased vitamin A absorption and storage, corroborating the present study.

Gonçalves et al. [13] found that the fat-soluble vitamins are absorbed in different sites in rats, specifically, that vitamin A is mostly absorbed in the proximal region, and vitamin $\mathrm{E}$, in the distal region of the intestine, and that vitamin $\mathrm{E}$ increased the absorption of vitamin A by $40 \%$. This finding in rats is in agreement with the finding of the present study in humans.

Vitamin E supplementation did not affect mature milk retinol twenty days after the intervention. Because of its unique composition, colostrum is rich in vitamin $\mathrm{A}$, but over time the composition of breast milk changes and its level of vitamin A decreases rapidly [37]. The present study found a significant decrease in breast milk retinol in both groups, confirming other literature reports [22,25,41]. 
Maternal supplementation with vitamin E was beneficial in the first days of breastfeeding but not later. A hypothesis to explain this finding would be that vitamin A and vitamin E may have supplied a maternal nutritional deficiency and from then on, the nutritional requirements of the newborn were not met. Based on the present study results, daily maternal supplementation with smaller doses of vitamins A and E after delivery could contribute to the proper statuses of these vitamins in the mother and newborn.

\section{Conclusions}

Maternal supplementation with vitamin E did not influence serum or mature milk retinol, but it increased colostrum vitamin A. Therefore, vitamin E improved the bioavailability of vitamin A in breast milk.

Acknowledgments: To School Maternity Hospital Januário Cicco and Unidade Mista das Quintas for having granted permission to conduct this study. To Nutrition Department UFRN and Postgraduate Program in Biochemistry-UFRN and Federal University of Rio Grande do Norte for supporting this study.

Author Contributions: J.F.S.D. and L.Q.L. idea, hypotheses, experimental design, wrote the manuscript, performed statistical evaluation. L.R.M.M., M.M.D., and N.L.N. data collection and analysis of samples. R.D. idea, experimental design, proofread the manuscript.

Conflicts of Interest: The authors declare no conflict of interest.

\section{References}

1. Berti, C.; Biesalski, H.K.; Gartner, R.; Lapillonne, A.; Pietrzik, K.; Poston, L.; Redman, C.; Koletzko, B.; Cetin, I. Micronutrients in pregnancy: Current knowledge and unresolved questions. Clin Nutr 2011, 30, 689-701.

2. Gerster, H. Vitamin a--functions, dietary requirements and safety in humans. Int J Vitam Nutr Res 1997, 67, 71-90.

3. Canete, A.; Cano, E.; Munoz-Chapuli, R.; Carmona, R. Role of vitamin a/retinoic acid in regulation of embryonic and adult hematopoiesis. Nutrients 2017, 9.

4. de Lira, L.Q.; Lima, M.S.; de Medeiros, J.M.; da Silva, I.F.; Dimenstein, R. Correlation of vitamin a nutritional status on alpha-tocopherol in the colostrum of lactating women. Matern Child Nutr 2013, 9, 31-40.

5. Grilo, E.C.; Lima, M.S.; Cunha, L.R.; Gurgel, C.S.; Clemente, H.A.; Dimenstein, R. Effect of maternal vitamin a supplementation on retinol concentration in colostrum. $J$ Pediatr (Rio J) 2015, 91, 81-86.

6. Rumbold, A.; Ota, E.; Hori, H.; Miyazaki, C.; Crowther, C.A. Vitamin e supplementation in pregnancy. Cochrane Database Syst Rev 2015, CD004069.

7. Bell, E.F.; Hansen, N.I.; Brion, L.P.; Ehrenkranz, R.A.; Kennedy, K.A.; Walsh, M.C.; Shankaran, S.; Acarregui, M.J.; Johnson, K.J.; Hale, E.C., et al. Serum tocopherol levels in 
very preterm infants after a single dose of vitamin e at birth. Pediatrics 2013, 132, e1626-1633.

8. Clemente, H.A.; Ramalho, H.M.; Lima, M.S.; Grilo, E.C.; Dimenstein, R. Maternal supplementation with natural or synthetic vitamin e and its levels in human colostrum. J Pediatr Gastroenterol Nutr 2015, 60, 533-537.

9. Brion, L.P.; Bell, E.F.; Raghuveer, T.S. Vitamin e supplementation for prevention of morbidity and mortality in preterm infants. Cochrane Database Syst Rev 2003, CD003665.

10. Lima, M.S.; Dimenstein, R.; Ribeiro, K.D. Vitamin e concentration in human milk and associated factors: A literature review. J Pediatr (Rio J) 2014, 90, 440-448.

11. Garcia, L.; Ribeiro, K.; Araujo, K.; Pires, J.; Azevedo, G.; Dimenstein, R. Alpha-tocopherol concentration in the colostrum of nursing women supplemented with retinyl palmitate and alpha-tocopherol. J Hum Nutr Diet 2010, 23, 529-534.

12. Ametaj, B.N.; Nonnecke, B.J.; Franklin, S.T.; Horst, R.L.; Bidlack, W.R.; Stuart, R.L.; Beitz, D.C. Dietary vitamin a modulates the concentrations of rrr-alpha-tocopherol in plasma lipoproteins from calves fed milk replacer. J Nutr 2000, 130, 629-636.

13. Goncalves, A.; Roi, S.; Nowicki, M.; Dhaussy, A.; Huertas, A.; Amiot, M.J.; Reboul, E. Fatsoluble vitamin intestinal absorption: Absorption sites in the intestine and interactions for absorption. Food Chem 2015, 172, 155-160.

14. Faul, F.; Erdfelder, E.; Lang, A.G.; Buchner, A. G*power 3: A flexible statistical power analysis program for the social, behavioral, and biomedical sciences. Behav Res Methods 2007, 39, 175-191.

15. Compounds, I.o.M.U.P.o.D.A.a.R. Dietary reference intakes for vitamin c, vitamin e, selenium, and carotenoids. In Washington (dc): National academies press (us), Washington (DC), 2000.

16. Ortega, R.M.; Lopez-Sobaler, A.M.; Martinez, R.M.; Andres, P.; Quintas, M.E. Influence of smoking on vitamin e status during the third trimester of pregnancy and on breastmilk tocopherol concentrations in spanish women. Am J Clin Nutr 1998, 68, 662-667.

17. Giuliano, A.R.; Neilson, E.M.; Kelly, B.E.; Canfield, L.M. Simultaneous quantitation and separation of carotenoids and retinol in human milk by high-performance liquid chromatography. Methods Enzymol 1992, 213, 391-399.

18. Nierenberg, D.W.; Nann, S.L. A method for determining concentrations of retinol, tocopherol, and five carotenoids in human plasma and tissue samples. Am J Clin Nutr 1992, 56, 417-426.

19. Milne, D.B.; Botnen, J. Retinol, alpha-tocopherol, lycopene, and alpha- and betacarotene simultaneously determined in plasma by isocratic liquid chromatography. Clin Chem 1986, 32, 874-876.

20. Organization, W.H. Global prevalence of vitamin a deficiency in populations at risk 19952005: Who global database on vitamin a deficiency. 2009.

21. West, K.P., Jr. Extent of vitamin a deficiency among preschool children and women of reproductive age. J Nutr 2002, 132, 2857S-2866S.

22. Macias, C.; Schweigert, F.J. Changes in the concentration of carotenoids, vitamin a, alpha-tocopherol and total lipids in human milk throughout early lactation. Ann Nutr Metab 2001, 45, 82-85.

23. Picciano, M.F. Pregnancy and lactation: Physiological adjustments, nutritional requirements and the role of dietary supplements. J Nutr 2003, 133, 1997S-2002S.

24. Gernand, A.D.; Schulze, K.J.; Stewart, C.P.; West, K.P., Jr.; Christian, P. Micronutrient deficiencies in pregnancy worldwide: Health effects and prevention. Nat Rev Endocrinol 2016, 12, 274-289.

25. Grilo, E.C.; Medeiros, W.F.; Silva, A.G.; Gurgel, C.S.; Ramalho, H.M.; Dimenstein, R. Maternal supplementation with a megadose of vitamin a reduces colostrum level of alpha-tocopherol: A randomised controlled trial. J Hum Nutr Diet 2016, 29, 652-661. 
26. Wang, Y.Z.; Ren, W.H.; Liao, W.Q.; Zhang, G.Y. Concentrations of antioxidant vitamins in maternal and cord serum and their effect on birth outcomes. J Nutr Sci Vitaminol (Tokyo) 2009, 55, 1-8.

27. Dimenstein, R.; Lira, L.; Medeiros, A.C.; Cunha, L.R.; Stamford, T.L. [effect of vitamin e supplementation on alpha-tocopherol levels in human colostrum]. Rev Panam Salud Publica 2011, 29, 399-403.

28. Rodríguez, G.P.; Alonso, D.P.; Sintes, G.S.; Matos, C.M.; Hernández, A.C.; Enríques, Y.; Gutiérrez, P.M. Vitaminas antioxidantes en un grupo de embarazadas y recién nacidos durante un año de estudio. Rev Cubana Aliment Nutr 2002, 16, 85-94.

29. Dimenstein, R.; Pires, J.F.; Garcia, L.R.; Lira, L.Q. [levels of alpha-tocopherol in maternal serum and colostrum of adolescents and adults]. Rev Bras Ginecol Obstet 2010, 32, 267272.

30. Fernandes, T.F.; Andreto, L.M.; Vieira, C.S.; de Arruda, I.K.; Diniz Ada, S. Serum retinol concentrations in mothers and newborns at delivery in a public maternity hospital in recife, northeast brazil. J Health Popul Nutr 2014, 32, 28-35.

31. Menon, K.C.; Skeaff, S.A.; Thomson, C.D.; Gray, A.R.; Ferguson, E.L.; Zodpey, S.; Saraf, A.; Das, P.K.; Toteja, G.S.; Pandav, C.S. Concurrent micronutrient deficiencies are prevalent in nonpregnant rural and tribal women from central india. Nutrition 2011, 27, 496-502.

32. Samba, C.; Tchibindat, F.; Gourmel, B.; Houze, P.; Malvy, D. Prevalence of vitamin a deficiency in pregnant and lactating women in the republic of congo. J Health Popul Nutr 2013, 31, 28-36.

33. Kaestel, P.; Martinussen, T.; Aaby, P.; Michaelsen, K.F.; Friis, H. Serum retinol is associated with stage of pregnancy and the acute phase response in pregnant women in guinea-bissau. J Nutr 2012, 142, 942-947.

34. Gebreselassie, S.G.; Gase, F.E.; Deressa, M.U. Prevalence and correlates of prenatal vitamin a deficiency in rural sidama, southern ethiopia. J Health Popul Nutr 2013, 31, 185-194.

35. Weber, D.; Stuetz, W.; Bernhard, W.; Franz, A.; Raith, M.; Grune, T.; Breusing, N. Oxidative stress markers and micronutrients in maternal and cord blood in relation to neonatal outcome. Eur J Clin Nutr 2014, 68, 215-222.

36. Yang, C.; Chen, J.; Liu, Z.; Yun, C.; Piao, J.; Yang, X. Prevalence and influence factors of vitamin a deficiency of chinese pregnant women. Nutr J 2016, 15, 12.

37. Schweigert, F.J.; Bathe, K.; Chen, F.; Buscher, U.; Dudenhausen, J.W. Effect of the stage of lactation in humans on carotenoid levels in milk, blood plasma and plasma lipoprotein fractions. Eur J Nutr 2004, 43, 39-44.

38. Marques, R.F.; Lopez, F.A.; Braga, J.A. [growth of exclusively breastfed infants in the first 6 months of life]. J Pediatr (Rio J) 2004, 80, 99-105.

39. Ribeiro, K.D.; Araujo, K.F.; Pereira, M.C.; Dimenstein, R. Evaluation of retinol levels in human colostrum in two samples collected at an interval of 24 hours. J Pediatr (Rio J) 2007, 83, 377-380.

40. Moore, T. The effect of vitamin e deficiency on the vitamin a reserves of the rat. Biochem J 1940, 34, 1321-1328.

41. Ross, J.S.; Harvey, P.W. Contribution of breastfeeding to vitamin a nutrition of infants: A simulation model. Bull World Health Organ 2003, 81, 80-86. 